\title{
Note on "Common fixed point theorem for weakly compatible multi-valued mappings satisfying strongly tangential property"
}

\author{
SAID BeLOUL
}

\begin{abstract}
In our paper [1], the existence and uniqueness of a common fixed point for multi valued mappings was given, hence in this note we point out to no uniqueness of common fixed point in theorem 3.1, corollary 3.1 and corollary 3.2 . We provide an example to demonstrate our purpose.
\end{abstract}

\section{MAin Results}

Theorem 1. Let $f, g: X \rightarrow X$, be single valued mappings and $S, T: X \rightarrow$ $C B(X)$ multi-valued mappings of a metric space $(X, d)$, such for all $x, y$ in $X$ we have:

(1)

$$
\begin{aligned}
\int_{0}^{H(S x, T y)} \varphi(t) \leq \phi & \left(\int_{0}^{d(f x, g y)} \varphi(t) \mathbf{d} t, \int_{0}^{d(f x, S x)} \varphi(t) \mathbf{d} t\right. \\
& \left.\int_{0}^{d(g y, T y)} \varphi(t) \mathbf{d} t, \int_{0}^{d(f x, T y)} \varphi(t) \mathbf{d} t, \int_{0}^{d(g y, S x)} \varphi(t) \mathbf{d} t\right),
\end{aligned}
$$

where $\phi: \mathbb{R}_{+}^{5} \rightarrow \mathbb{R}_{+}$is an upper semi continuous, non decreasing function satisfying:

$$
\psi(t)=\max (\phi(0, t, 0,0, t), \phi(0,0, t, t, 0), \phi(t, 0,0, t, t))<t,
$$

for any $t>0$ and $\varphi: \mathbb{R}_{+} \rightarrow \mathbb{R}_{+}$is a Lebesgue-integrable function which is summable on each compact subset of $\mathbb{R}_{+}$, non-negative, and such that for each $\varepsilon>0, \int_{0}^{\varepsilon} \varphi(t) \mathbf{d} t>0$. Suppose that the two pairs $(f, S),(g, T)$ are weakly compatible, moreover if $(f, g)$ is strongly tangential w.r.t $(S, T)$, then $f, g, S$ and $T$ have a common fixed point in $X$.

2010 Mathematics Subject Classification. 47H10, 54H25.

Key words and phrases. Common fixed point, strongly tangential, weakly compatible, multi-valued maps. 
Remark 1. In paper [1], the proof of uniqueness of the common fixed point is false, since the inequality $d(a, b) \leq H(A, B)$, for all $a \in A$ and $b \in B$ is note true in general. For example $A=[0,2], B=[3,5]$ and $H(A, B)=3$, but $d(0,5)=5$ (with euclidian metric).

If $S=T$ and $f=g$, we obtain the following corollary:

Corollary 1. Let $f: X \rightarrow X$, and $S: X \rightarrow C B(X)$ be single and set valued mappings of metric space $(X, d)$ such:

$$
\begin{aligned}
\int_{0}^{H(S x, S y)} \varphi(t) \leq \phi & \left(\int_{0}^{d(f x, f y)} \varphi(t) \mathbf{d} t, \int_{0}^{d(f x, S x)} \varphi(t) \mathbf{d} t\right. \\
& \left.\int_{0}^{d(f y, S y)} \varphi(t) \mathbf{d} t, \int_{0}^{d(f x, S y)} \varphi(t) \mathbf{d} t, \int_{0}^{d(f y, S x)} \varphi(t) \mathbf{d} t\right),
\end{aligned}
$$

where $\phi \in \Phi$ and $\varphi: \mathbb{R}_{+} \rightarrow \mathbb{R}_{+}$is a Lebesgue-integrable function which is summable on each compact subset of $\mathbb{R}_{+}$, non-negative, and such that for each $\varepsilon>0, \int_{0}^{\varepsilon} \varphi(t) d t>0$, if $f$ is strongly tangential w.r.t $S$ and $(f, S)$ is weakly compatible, then $f$ and $S$ have a common fixed point.

Corollary 2. Let $f, g: X \rightarrow X$, and $S, T: X \rightarrow C B(X)$ be single and set valued mappings of metric space $(X, d)$ such:

$$
\begin{aligned}
\left(\int_{0}^{H(S x, T y)} \varphi(t) \mathbf{d} t\right)^{p} \leq a & \left(\int_{0}^{d(f x, g y)} \varphi(t) \mathbf{d} t\right)^{p}+b\left(\int_{0}^{d(f x, S x)} \varphi(t) \mathbf{d} t\right)^{p} \\
& +c\left(\int_{0}^{d(g y, T y)} \varphi(t) \mathbf{d} t\right)^{p},
\end{aligned}
$$

where $\phi \in \Phi$ and $\varphi: \mathbb{R}_{+} \rightarrow \mathbb{R}_{+}$is a Lebesgue-integrable function which is summable on each compact subset of $\mathbb{R}^{+}$, non-negative, and such that for each $\varepsilon>0, \int_{0}^{\varepsilon} \varphi(t) d t>0$. and $a, b, c$ are nonnegative real numbers such $a+b+b<1$ and $p \in \mathbb{N}^{\star}$, if $\{f, g\}$ is strongly tangential w.r.t $(S, T)$ and the two pairs $(f, g),(S, T)$ are weakly compatible, then $f, g, S$ and $T$ have a common fixed point.

Example 1. Let $X=[0,4]$ endowed with the euclidian metric. Define the mappings $f, g, S$ and $T$ as follows:

$$
x=g x=\left\{\begin{array}{ll}
\frac{x+1}{2}, & 0 \leq x \leq 1 ; \\
2, & 1<x \leq 2 ; \\
\frac{7}{2}, & 2<x \leq 4 ;
\end{array} \quad S x=T x= \begin{cases}\{1\}, & 0 \leq x<2 ; \\
\{2\}, & 2 \leq x \leq 4 .\end{cases}\right.
$$

Let $\left\{x_{n}\right\},\left\{y_{n}\right\}$ be two sequences which defined by: $x_{n}=1-e^{-n}$ and $y_{n}=$ $1-\frac{1}{n}$. Clearly that

$$
\lim _{n \rightarrow \infty} S x_{n}=\lim _{n \rightarrow \infty} S y_{n}=[0,1]
$$


and

$$
\lim _{n \rightarrow \infty} f x_{n}=\lim _{n \rightarrow \infty} f y_{n}=1 \in[0,1],
$$

then $f$ is strongly tangential w.r.t $S$. We will utilize Corollary 1 , with

$$
\phi\left(t_{1}, t_{2}, t_{3}, t_{4}, t_{5}\right)=\frac{2}{5} \max \left\{t_{1}, t_{2}, t_{3}, t_{4}, t_{5}\right\} \quad \text { and } \quad \varphi(t)=1 .
$$

For the contractive condition (1), we have the following cases:

(1) For $x, y \in[0,2) \cup[2,4]$, we have $H(S x, T y)=0$, so obviously that (1) is satisfied.

(2) For $x \in[0,2)$ and $y \in[2,4]$ we have

$$
H(S x, T y)=1 \leq \frac{7}{5}=\frac{2}{5} d(f y, S y) .
$$

(3) For $2 \leq x \leq 4$ and $y \in[0,2)$, we have

$$
H(S x, T y)=1 \leq \frac{7}{5}=\frac{2}{5} d(f x, S x) .
$$

Also 1 and 2 are two coincidence points satisfying $f S 1=[0,1]=S f 1$, $f S 2=\{2\}=S f 2$, consequently all hypotheses of Corollary 1 are satisfied, then $f$ and $S$ have at least a common fixed point, therefore 1 and 2 are two common fixed point.

\section{REFERENCES}

[1] S. Beloul, A Common Fixed Point Theorem for Weakly Compatible Multi-Valued Mappings Satisfying Strongly Tangential Property, Mathematica Moravica, 18-2 (2014), 63-72.

\section{Said Beloul}

DEPARTMENT OF MATHEMATICS

UNIVERSiTy OF ElOUED

P.O. Box 789 Eloued

Algeria

E-mail address: beloulsaid@gmail.com 